\title{
REPRESENTATION DIMENSION AS A RELATIVE HOMOLOGICAL INVARIANT OF STABLE EQUIVALENCE
}

\author{
ALEX S. DUGAS
}

\begin{abstract}
Over an Artin algebra $\Lambda$ many standard concepts from homological algebra can be relativized with respect to a contravariantly finite subcategory $\mathcal{C}$ of $\bmod -\Lambda$, which contains the projective modules. The main aim of this article is to prove that the resulting relative homological dimensions of modules are preserved by stable equivalences between Artin algebras. As a corollary, we see that Auslander's notion of representation dimension is invariant under stable equivalence (a result recently obtained independently by Guo). We then apply these results to the syzygy functor for self-injective algebras of representation dimension three, where we bound the number of simple modules in terms of the number of indecomposable nonprojective summands of an Auslander generator.
\end{abstract}

\section{Introduction AND Notation}

It has long been of interest to determine to what extent various natural invariants of Artin algebras are preserved by stable equivalences. Of particular importance are homological invariants, and especially those defined in terms of projective modules. Yet, even the simplest examples of stable equivalence (for instance, between radical square zero and hereditary algebras) already show that global and projective dimensions are not stable invariants. Nevertheless, it is established in [10] that projective dimensions of modules are preserved by stable equivalences between algebras without nodes. Our main result extends this fact to relative homological dimensions, defined with respect to contravariantly finite subcategories. In $\S 3$ we show that, given a stable equivalence $\alpha$ between algebras $\Lambda$ and $\Lambda^{\prime}$ without nodes, the relative projective dimension of $X$ with respect to a contravariantly finite subcategory $\mathcal{C}$ containing proj- $\Lambda$ equals the relative projective dimension of $\alpha X$ with respect to a corresponding contravariantly finite subcategory of mod- $\Lambda^{\prime}$. Next we look to stable equivalences given by separations of nodes, as defined in [9]. In this case we are able to prove analogous results, provided that the contravariantly finite subcategory $\mathcal{C}$ contains the nodes that are being separated. In particular, we obtain some insight into what is behind the failure of certain stable equivalences to preserve projective dimension. Furthermore, in both situations we obtain generalizations of the well-known result from [3] and [10] that stable equivalences commute with the syzygy operator on modules, showing that they commute with suitable relative syzygy operators as well.

One particularly nice consequence of these results is that Auslander's notion of representation dimension for Artin algebras [1] is invariant under stable equivalence. Our proof of this fact, presented in $\S 3$ and $\S 4$, makes use of a well-known relative homological characterization of representation dimension. This result, recently proven independently by Guo [7], as well as by Reiten and Saorin for self-injective algebras (unpublished), was the original motivation behind this article. Naturally, there are some similarities at the core of certain proofs, but the presentation here is substantially different, and we show that a wealth of relative homological information is preserved by stable equivalences for the same reasons that representation dimension is. 
In the fifth section, our intention is to show how such relative homological invariants may be related to the number of nonprojective simple $\Lambda$-modules. The case where $\Lambda$ is a self-injective algebra of representation dimension three turns out to be especially conducive to our methods. In particular, applying our results to the syzygy functor in this case, we are able to improve upon a result of Rouquier [12] relating the number of simple $\Lambda$-modules to the number of nonprojective indecomposable summands of an Auslander generator for $\Lambda$. As we shall see, the minimal number of nonprojective indecomposable summands of an Auslander generator is invariant under stable equivalence, and hence it is desirable to understand any relation that may exist between this number and the number of simple $\Lambda$-modules. Persuing these ideas we are led to some interesting structural restrictions on algebras having Auslander generators with additional properties. In our final section, we apply such results to help construct a specific Auslander generator over a self-injective special biserial algebra. In fact, we hereby see how these results also provide some useful information about the make-up of Auslander generators for algebras of representation dimension three.

Throughout this article, $R$ will be a commutative Artinian ring, and $\Lambda$ and $\Lambda^{\prime}$ will denote a pair of stably equivalent, basic Artin $R$-algebras with no semisimple blocks. We shall denote by mod- $\Lambda$ the category of finitely generated right $\Lambda$-modules, by mod- $\Lambda$ the stable module category, and by $\bmod _{\mathcal{P}} \Lambda$ the full subcategory of mod- $\Lambda$ consisting of modules with no projective direct summands. If $\alpha: \underline{\bmod }-\Lambda \rightarrow \underline{\bmod }-\Lambda^{\prime}$ is an equivalence, we shall also use $\alpha$ to denote the induced map mod- $\Lambda \rightarrow \bmod -\Lambda^{\prime}$ which takes projectives to zero. Furthermore, we shall write $\bar{\alpha}: \underline{\bmod }(\bmod -\Lambda) \rightarrow \underline{\bmod }\left(\bmod -\Lambda^{\prime}\right)$ for the induced equivalence of functor categories as in $[2]$ or $[3]$, where $\bmod (\bmod -\Lambda)$ denotes the abelian category consisting of finitely presented, contravariant, additive functors from mod- $\Lambda$ to the category of abelian groups, which vanish on $\Lambda$. We shall abbreviate the groups $\operatorname{Hom}_{\Lambda}(A, B)$ as $(A, B)$ when there is no ambiguity regarding the ring $\Lambda$. We denote the morphism sets in mod- $\Lambda$ by $\underline{\operatorname{Hom}}_{\Lambda}(A, B)$, which we sometimes abbreviate as $(\underline{A}, \underline{B})$. If $f \in(A, B), \underline{f}$ will denote the image of $f$ in $\underline{\operatorname{Hom}}_{\Lambda}(A, B)$. Finally, all functors will be assumed to be additive and $R$ linear, and all subcategories will be assumed to be full, additive $R$-subcategories, closed under isomorphisms and direct summands, unless noted otherwise.

\section{Stable Equivalence and Exact Sequences}

We begin by studying the effect of a stable equivalence $\alpha$ on short exact sequences. Much of these ideas originated in the work of Auslander and Reiten in [3] and were later extended by Martínez Villa in [10]. The proof of our main result relies heavily on Theorem 1.7 from [10], which includes the assumption that the algebras have no nodes (see $\S 4$ for the definition of a node). Hence, throughout this section and the next we shall assume that $\Lambda$ and $\Lambda^{\prime}$ have no nodes, unless stated otherwise. We then consider stable equivalences between algebras with nodes in $\S 4$. Before stating Martínez Villa's theorem, we quickly recall one other result from the same paper.

Proposition 2.1. Let $P$ be an indecomposable, noninjective projective $\Lambda$-module. Then $\bar{\alpha} \operatorname{Ext}_{\Lambda}^{1}(-, P) \cong \operatorname{Ext}_{\Lambda^{\prime}}^{1}\left(-, P^{\prime}\right)$ for some indecomposable, noninjective projective $\Lambda^{\prime}$-module $P^{\prime}$.

Following [10] we shall denote the $\Lambda^{\prime}$-module $P^{\prime}$ by $\alpha^{\prime} P$, and we extend $\alpha^{\prime}$ additively to all projective modules with no injective summands. In fact, $\alpha^{\prime}$ gives a bijection between the indecomposable noninjective projective modules over $\Lambda$ and $\Lambda^{\prime}$. Finally, recall that a short exact sequence is said to be minimal if it contains no nonzero split exact sequence as a direct summand. 
Theorem 2.2 (Martínez Villa [10]). Let $\alpha: \underline{\bmod }-\Lambda \rightarrow \underline{\bmod }-\Lambda^{\prime}$ be a stable equivalence between two algebras with no nodes and no semisimple blocks. Let

$$
0 \rightarrow A \oplus Q_{1} \stackrel{f}{\longrightarrow} B \oplus P \oplus Q_{2} \stackrel{g}{\longrightarrow} C \rightarrow 0
$$

be a minimal short exact sequence in $\bmod -\Lambda$ where $A, B, C \in \bmod _{\mathcal{P}} \Lambda, Q_{1}, Q_{2}$ are projective modules with no injective summand, and $P$ is projective and injective. Then there exists a minimal short exact sequence

$$
0 \rightarrow \alpha A \oplus \alpha^{\prime} Q_{1} \stackrel{u}{\longrightarrow} \alpha B \oplus \alpha^{\prime} Q_{2} \oplus P^{\prime} \stackrel{v}{\longrightarrow} \alpha C \rightarrow 0
$$

in $\bmod -\Lambda^{\prime}$ with $P^{\prime}$ projective injective, and $\underline{v}=\alpha \underline{g}$.

We now proceed to develop a couple of useful corollaries to the above theorem. To begin with, we show that we can basically drop the minimality conditions.

Corollary 2.3. Let all notation and hypotheses be as in the previous theorem, except we do not assume that the given short exact sequence of $\Lambda$-modules is minimal. Then there exists a (not necessarily minimal) short exact sequence of $\Lambda^{\prime}$-modules as in the theorem.

Proof. If the given short exact sequence is not minimal we may write it as the direct sum of a minimal short exact sequence and a split short exact sequence which, up to isomorphism, has the form $0 \rightarrow A^{\prime} \longrightarrow A^{\prime} \oplus C^{\prime} \longrightarrow C^{\prime} \rightarrow 0$ where $A^{\prime}$ and $C^{\prime}$ are summands of $A \oplus Q_{1}$ and $C$ respectively. Applying the theorem to the minimal sequence yields a minimal sequence over $\Lambda^{\prime}$. Meanwhile, since $A \oplus Q_{1}$ and $C$ have no projective injective summands, applying $\alpha$ and $\alpha^{\prime}$ directly to the split sequence will yield a split exact sequence over $\Lambda^{\prime}$. The additivity of $\alpha$ and $\alpha^{\prime}$ clearly implies that the direct sum of these two short exact sequences of $\Lambda^{\prime}$-modules has all the desired properties.

Next, turning our attention to long exact sequences, we obtain the following corollary by decomposing an exact sequence into short exact sequences, applying the above corollary, and splicing together the resulting short exact sequences over $\Lambda^{\prime}$.

Corollary 2.4. Suppose

$$
\cdots \rightarrow C_{i} \oplus P_{i} \oplus Q_{i} \stackrel{f_{i}}{\longrightarrow} C_{i+1} \oplus P_{i+1} \oplus Q_{i+1} \stackrel{f_{i+1}}{\longrightarrow} C_{i+2} \oplus P_{i+2} \oplus Q_{i+2} \rightarrow \cdots
$$

is an exact sequence of $\Lambda$-modules such that for each $i, C_{i}$ belongs to $\bmod _{\mathcal{P}} \Lambda, P_{i}$ is projective injective, $Q_{i}$ is projective with no injective summands, and ker $f_{i}$ has no projective injective summands. Then there exists an exact sequence of $\Lambda^{\prime}$-modules

$$
\cdots \rightarrow \alpha C_{i} \oplus P_{i}^{\prime} \oplus \alpha^{\prime} Q_{i} \stackrel{f_{i}^{\prime}}{\longrightarrow} \alpha C_{i+1} \oplus P_{i+1}^{\prime} \oplus \alpha^{\prime} Q_{i+1} \stackrel{f_{i+1}^{\prime}}{\longrightarrow} \alpha C_{i+2} \oplus P_{i+2}^{\prime} \oplus \alpha^{\prime} Q_{i+2} \rightarrow \cdots
$$

such that for each $i, P_{i}^{\prime}$ is projective injective, and ker $f_{i}^{\prime}$ contains no projective injective summands. Moreover, the $i^{\text {th }}$ term of this sequence may be taken to be zero whenever the $i^{\text {th }}$ term of the given sequence is zero.

Proof. Let ker $f_{i}=K_{i} \oplus Q_{i}^{\prime}$ for $K_{i} \in \bmod _{\mathcal{P}} \Lambda$ and $Q_{i}^{\prime}$ projective with no injective summands. Then, for each $i$, we have a short exact sequence

$$
0 \rightarrow K_{i} \oplus Q_{i}^{\prime} \longrightarrow C_{i} \oplus P_{i} \oplus Q_{i} \longrightarrow K_{i+1} \oplus Q_{i+1}^{\prime} \rightarrow 0
$$

which, since $Q_{i+1}^{\prime}$ is projective, contains a sequence isomorphic to $0 \rightarrow 0 \longrightarrow Q_{i+1}^{\prime} \stackrel{I d}{\longrightarrow}$ $Q_{i+1}^{\prime} \rightarrow 0$ as a direct summand. Applying the previous corollary to the complement to this 
sequence, and taking the direct sum of the result with $0 \rightarrow 0 \longrightarrow \alpha^{\prime} Q_{i+1}^{\prime} \stackrel{I d}{\longrightarrow} \alpha^{\prime} Q_{i+1}^{\prime} \rightarrow 0$ now yields the short exact sequence

$$
0 \rightarrow \alpha K_{i} \oplus \alpha^{\prime} Q_{i}^{\prime} \longrightarrow \alpha C_{i} \oplus P_{i}^{\prime} \oplus \alpha^{\prime} Q_{i} \longrightarrow \alpha K_{i+1} \oplus \alpha^{\prime} Q_{i+1}^{\prime} \rightarrow 0 .
$$

Finally, splicing these together produces a long exact sequence in mod- $\Lambda^{\prime}$ of the desired form.

Remark. Notice that, up to homotopy, any exact sequence of $\Lambda$-modules is isomorphic to one of the above form. For, any projective injective direct summand $P$ of ker $f_{i}$ must split off as a summand of both $P_{i}$ and $P_{i+1}$ and hence the given exact sequence has as a summand the null-homotopic sequence

$$
\cdots \rightarrow 0 \rightarrow P \stackrel{I d}{\longrightarrow} P \rightarrow 0 \rightarrow \cdots
$$

where the $P$ 's lie in degrees $i$ and $i+1$.

\section{Relative Homological Algebra and Representation Dimension}

For motivation, we begin by reviewing Auslander's notion of representation dimension of an algebra. We shall define the representation dimension of $\Lambda$ as

$$
\text { rep.dim } \Lambda=\inf \left\{\text { gl.dim } \operatorname{End}_{\Lambda}(M) \mid M \text { is a generator-cogenerator for } \Lambda\right\},
$$

which differs from Auslander's original definition only for semisimple algebras. Any generatorcogenerator $M$ for which this infimum is attained is called an Auslander generator. As long as $\Lambda$ is not semisimple, the representation dimension of $\Lambda$ admits the following relative homological characterization. Namely, rep.dim $\Lambda \leq n(n \geq 2)$ if and only if there exists a generator-cogenerator $M$ such that for every indecomposable $\Lambda$-module $X$ there exists an exact sequence

$$
0 \rightarrow M_{n-2} \stackrel{f_{n-2}}{\longrightarrow} M_{n-3} \rightarrow \cdots \rightarrow M_{0} \stackrel{f_{0}}{\longrightarrow} X \rightarrow 0
$$

with each $M_{i} \in \operatorname{add}(M)$ and with the property that the induced sequence

$$
0 \rightarrow\left(M, M_{n-2}\right) \stackrel{\left(M, f_{n-2}\right)}{\longrightarrow} \cdots \rightarrow\left(M, M_{0}\right) \stackrel{\left(M, f_{0}\right)}{\longrightarrow}(M, X) \rightarrow 0
$$

is also exact. It is easily seen that this condition, for any generator-cogenerator $M$, is equivalent to its own dual, as well as to gl.dim $\operatorname{End}_{\Lambda}(M) \leq n$ (see [1] or [6] for details).

In view of (3.2), the sequence (3.1) is a relative add $(M)$-resolution for $X$. More generally, we can replace add $(M)$ with a fixed contravariantly finite subcategory $\mathcal{C}$ of mod- $\Lambda$ which contains proj- $\Lambda$. We will henceforth refer to a subcategory containing proj- $\Lambda$ as a generator subcategory. (Of course, we could obtain dual results by considering covariantly finite cogenerator subcategories, i.e., containing all the injective $\Lambda$-modules, but we leave such formulations to the reader.) We will later be able to recover results about representation dimension by specializing to the case $\mathcal{C}=\operatorname{add}(M)$ for an Auslander generator $M$, noting that $\operatorname{add}(M)$ is contravariantly finite since it is of finite type.

We now review some important notions from relative homological algebra. Many of the following ideas appear also in [5], phrased in terms of subfunctors of $\operatorname{Ext}_{\Lambda}^{1}(-,-)$ with enough projectives. Since $\mathcal{C}$ is contravariantly finite, any $\Lambda$-module $X$ admits a $\mathcal{C}$-resolution

$$
\cdots \rightarrow C_{1} \stackrel{f_{1}}{\longrightarrow} C_{0} \stackrel{f_{0}}{\longrightarrow} X \rightarrow 0
$$


where the induced sequence

$$
\cdots \rightarrow\left(C, C_{1}\right) \stackrel{\left(C, f_{1}\right)}{\longrightarrow}\left(C, C_{0}\right) \stackrel{\left(C, f_{0}\right)}{\longrightarrow}(C, X) \rightarrow 0
$$

is exact for all $C \in \mathcal{C}$. To construct such a resolution, start by taking a right $\mathcal{C}$-approximation $f_{0}: C_{0} \rightarrow X$ of $X$ and continue by taking $f_{i}: C_{i} \rightarrow \operatorname{ker} f_{i-1}$ to be a right $\mathcal{C}$-approximation for each $i$. Since proj- $\Lambda \subseteq \mathcal{C}$, each right $\mathcal{C}$-approximation is an epimorphism and the exactness of the resolution follows. If each $f_{i}$ is taken to be a minimal right $\mathcal{C}$-approximation, we obtain a minimal $\mathcal{C}$-resolution of $X$. Since minimal right $\mathcal{C}$-approximations are unique up to isomorphism, so are minimal $\mathcal{C}$-resolutions, and thus we may define $\Omega_{\mathcal{C}}^{n} X=\operatorname{ker} f_{n-1}$ for $n>0$ in a minimal $\mathcal{C}$-resolution of $X$ as above.

Much of the standard theory for projective resolutions can be generalized to this setting. In particular, a $\mathcal{C}$-resolution of $X$ is unique up to homotopy, and this allows us to define derived functors of $(-, Y)$ relative to $\mathcal{C}$. We write $\operatorname{Ext}_{\mathcal{C}}^{i}(X, Y)=H^{i}\left(C_{*}, Y\right)$ where $C_{*}$ denotes a $\mathcal{C}$-resolution of $X$. We also obtain a relative version of projective dimension, $\mathcal{C}$-dimension, defined by $\mathcal{C}-\operatorname{dim} X=\inf \left\{n \geq 0 \mid \Omega_{\mathcal{C}}^{n+1} X=0\right\}$. As with projective dimension, one can prove the following.

Lemma 3.1. For a $\Lambda$-module $X$, the following are equivalent:

(1) $\mathcal{C}-\operatorname{dim} X \leq n$.

(2) The minimal $\mathcal{C}$-resolution of $X$ has length at most $n$.

(3) $X$ has a $\mathcal{C}$-resolution

$$
0 \rightarrow C_{n} \rightarrow \cdots \rightarrow C_{1} \longrightarrow C_{0} \longrightarrow X \rightarrow 0 .
$$

(4) $\operatorname{Ext}_{\mathcal{C}}^{i}(X, Y)=0$ for all $i>n$ and all $\Lambda$-modules $Y$.

When $\mathcal{C}=\operatorname{add}(M)$ for a generator $M$, we shall simplify our notation a bit by writing $M$, instead of $\operatorname{add}(M)$, wherever we have included $\mathcal{C}$ as part of a relativized definition above. For instance, in the discussion of representation dimension at the beginning of this section, (3.1) is an $M$-resolution of $X$ giving $M$-dim $X \leq n-2$. Thus, for $n \geq 2$, rep.dim $\Lambda \leq n$ if and only if there exists a generator-cogenerator $M$ such that $M$-dim $X \leq n-2$ for every $\Lambda$-module $X$.

Now let $X$ be an indecomposable nonprojective $\Lambda$-module with a minimal $\mathcal{C}$-resolution

$$
\cdots \rightarrow M_{r} \stackrel{f_{r}}{\longrightarrow} M_{r-1} \rightarrow \cdots \rightarrow M_{0} \stackrel{f_{0}}{\longrightarrow} X \rightarrow 0 .
$$

Since each $f_{i}$ is right minimal, ker $f_{i}$ contains no summands of $M_{i}$ and hence no projective injective summands for every $i$. Thus, Corollary 2.4 yields an exact sequence

$$
\cdots \rightarrow N_{r} \stackrel{g_{r}}{\longrightarrow} N_{r-1} \rightarrow \cdots \rightarrow N_{0} \stackrel{g_{0}}{\longrightarrow} \alpha X \rightarrow 0 .
$$

Let $\alpha \mathcal{C}$ denote the full subcategory of $\bmod -\Lambda^{\prime}$ additively generated by all projective modules and all modules isomorphic to $\alpha C$ for some nonprojective $C \in \mathcal{C}$. Clearly each $N_{i} \in \alpha \mathcal{C}$, and we wish to show that (3.3) is in fact an $\alpha \mathcal{C}$-resolution of $\alpha X$. In view of the proof of Corollary 2.4, it suffices to check that $\alpha$, in an appropriate sense (see below), takes right $\mathcal{C}$-approximations to right $\alpha \mathcal{C}$-approximations. We first prove a simple lemma.

Lemma 3.2. Let $\Lambda$ be any Artin algebra (possibly with nodes), and let $g: B \rightarrow C$ be an epimorphism such that $(\underline{X}, \underline{g}):(\underline{X}, \underline{B}) \rightarrow(\underline{X}, \underline{C})$ is surjective for some $\Lambda$-module $X$. Then $(X, g):(X, B) \rightarrow(X, C)$ is also surjective.

Proof. Suppose we have a map $s: X \rightarrow C$. Then there exists a map $t: X \rightarrow B$ such that $g t=\underline{s}$. Thus $s-g t: X \rightarrow C$ factors through the projective cover $\pi: P_{C} \rightarrow C$, say $s-g \bar{t}=\pi u$ for some map $u: X \rightarrow P_{C}$. Clearly $\pi$ factors through the epimorphism $g$, so 
we have $\pi=g w$ for a map $w: P_{C} \rightarrow B$. Hence, $s=g t+\pi u=g t+g w u=g(t+w u)$ and $s$ factors through $g$ as required.

Corollary 3.3. For any Artin algebra $\Lambda$ and any generator subcategory $\mathcal{C} \subseteq \bmod -\Lambda$, let $g: C \rightarrow X$ be a right $\mathcal{C}$-approximation. If $v: \alpha C \oplus P \rightarrow \alpha X \oplus Q$ is an epimorphism with $P$ and $Q$ projective such that $\underline{v}=\alpha g$, then $v$ is a right $\alpha \mathcal{C}$-approximation. In particular, if $\mathcal{C}$ is contravariantly finite in $\bmod -\Lambda^{-}$then so is $\alpha \mathcal{C}$ in $\bmod -\Lambda^{\prime}$.

Proof. Since $\mathcal{C}$ contains the projectives, $g$ must be an epimorphism, and hence for any $C^{\prime} \in \mathcal{C},\left(C^{\prime}, g\right)$ and $\left(\underline{C}^{\prime}, \underline{g}\right)$ are surjective. Applying $\alpha$ shows that $\left(\underline{\alpha C^{\prime}}, \underline{v}\right)$ is surjective. The above lemma now implies that $\left(\alpha C^{\prime}, v\right)$ is surjective. Since $\left(\Lambda^{\prime}, v\right)$ is clearly surjective too, $v$ is a right $\alpha \mathcal{C}$-approximation.

Notice that this corollary applies directly to the situation found in Theorem 2.2, and it follows immediately that the sequence (3.3) is constructed from short exact sequences which are right $\alpha \mathcal{C}$-approximations. Hence, it is an $\alpha \mathcal{C}$-resolution. We now summarize these results in the following theorem.

Theorem 3.4. Let $\alpha: \underline{\bmod }-\Lambda \rightarrow \bmod -\Lambda^{\prime}$ be a stable equivalence between two Artin $R$ algebras without nodes, let $\mathcal{C}$ be a contravariantly finite generator subcategory of mod- $\Lambda$, and let $X$ be an indecomposable, nonprojective $\Lambda$-module. Then

(a) If $\cdots \rightarrow M_{1}^{\prime} \oplus P_{1} \oplus Q_{1} \stackrel{f_{1}}{\longrightarrow} M_{0}^{\prime} \oplus P_{0} \oplus Q_{0} \stackrel{f_{0}}{\longrightarrow} X \rightarrow 0$ is a $\mathcal{C}$-resolution of $X$ with $M_{i}^{\prime}$ in $\bmod _{\mathcal{P}} \Lambda, Q_{i}$ projective with no injective summands, and $P_{i}$ projective injective for each $i$, then $\alpha X$ has an $\alpha \mathcal{C}$-resolution

$$
\cdots \rightarrow \alpha M_{1}^{\prime} \oplus P_{1}^{\prime} \oplus \alpha^{\prime} Q_{1} \stackrel{g_{1}}{\longrightarrow} \alpha M_{0}^{\prime} \oplus P_{0}^{\prime} \oplus \alpha^{\prime} Q_{0} \stackrel{g_{0}}{\longrightarrow} \alpha X \rightarrow 0
$$

where $P_{i}^{\prime}$ is projective injective for each $i$. Moreover, these two resolutions have equal lengths.

(b) If $\Omega_{\mathcal{C}} X \cong Y \oplus Q$ for $Y \in \bmod _{\mathcal{P}} \Lambda$ and $Q$ projective with no injective summands, then $\Omega_{\alpha \mathcal{C}}(\alpha X) \cong \alpha Y \oplus \alpha^{\prime} Q$.

(c) $\mathcal{C}$-dim $X=\alpha \mathcal{C}$-dim $\alpha X$.

Proof. Part (a) follows from the above arguments, while Corollary 2.4 yields the statement about the lengths of the resolutions. Part (b) is obvious if $X$ belongs to $\mathcal{C}$, and otherwise it follows from Theorem 2.2 applied to the minimal short exact sequence $0 \rightarrow \Omega_{\mathcal{C}} X \longrightarrow C \stackrel{f}{\longrightarrow}$ $X \rightarrow 0$ where $f$ is a minimal right $\mathcal{C}$-approximation, together with Corollary 3.3. Part (c) is now a simple consequence of the definition of $\mathcal{C}$-dimension.

Corollary 3.5. If $\alpha$ : $\bmod -\Lambda \rightarrow \bmod -\Lambda^{\prime}$ is a stable equivalence between two Artin $R$-algebras without nodes, then rep.dim $\Lambda=$ rep.dim $\Lambda^{\prime}$. Moreover, if $M=M^{\prime} \oplus P$ is an Auslander generator for $\Lambda$ with $M^{\prime} \in \bmod _{\mathcal{P}} \Lambda$ and $P$ projective, then $\alpha M^{\prime} \oplus \Lambda^{\prime}$ is an Auslander generator for $\Lambda^{\prime}$.

Proof. Let $\mathcal{C}=\operatorname{add}(M)$ and apply part (c) of the above theorem. The only thing that remains to be checked is that $\alpha M^{\prime} \oplus \Lambda^{\prime}$ is a cogenerator for $\Lambda^{\prime}$. If $I^{\prime}$ is an indecomposable, nonprojective injective $\Lambda^{\prime}$-module, we claim that $I^{\prime} \cong \alpha I$ for some indecomposable, nonprojective injective $\Lambda$-module $I$. Since $I \in \operatorname{add}\left(M^{\prime}\right)$, we then have $I^{\prime} \in \operatorname{add}\left(\alpha M^{\prime}\right)$ and the result follows. To verify the above claim, suppose $I=\alpha^{-1} I^{\prime}$ is not injective. Then, by Lemma 3.4 of [3], $\operatorname{Ext}_{\Lambda^{\prime}}^{1}\left(-, I^{\prime}\right) \cong \bar{\alpha} \operatorname{Ext}_{\Lambda}^{1}(-, I) \neq 0$, contradicting the injectivity of $I^{\prime}$. The 
final statement holds since $\alpha \mathcal{C}=\operatorname{add}\left(\alpha M^{\prime} \oplus \Lambda^{\prime}\right)$.

Corollary 3.6. If $\Lambda$ has no nodes, the minimal number of nonprojective indecomposable summands in an Auslander generator for $\Lambda$ is an invariant of the stable category mod- $\Lambda$.

In light of these results it would be interesting to find an equivalent definition of representation dimension solely in terms of the stable category mod- $\Lambda$.

\section{Separation of Nodes}

In this section, we investigate when relative homological dimensions are preserved by stable equivalences given by separations of nodes, as defined in [9]. As a result, we will see that such stable equivalences also preserve representation dimension. Together with Martínez Villa's work showing that any algebra is stably equivalent, via a separation of nodes, to an algebra without nodes, this result completes our proof that any two stably equivalent Artin algebras have equal representation dimension.

Our approach to the problem in this case is essentially the same as in the preceding sections. Unfortunately, much of the theory of short exact sequences under stable equivalences becomes invalid in the presence of nodes, and thus we shall need to develop ad hoc analogues of such results. Nevertheless, our explicit knowledge of a functor providing the stable equivalence in this case simplifies matters greatly. We begin by describing this functor and the process of separating nodes from [9].

A nonprojective, noninjective simple $\Lambda$-module $S$ is called a node of $\Lambda$ if the middle term of the almost split sequence starting in $S$ is projective. Let $S=S_{1} \oplus \cdots \oplus S_{n}$ be a sum of nonisomorphic nodes, let $\mathfrak{a}=\tau_{\Lambda}(S)$ be the trace ideal of $S$ in $\Lambda$, and let $\mathfrak{b}=\operatorname{ann}_{r}(\mathfrak{a})$ be the right annihilator of $\mathfrak{a}$ in $\Lambda$. Define $\Gamma$ to be the triangular matrix ring

$$
\Gamma=\left(\begin{array}{cc}
\Lambda / \mathfrak{a} & \mathfrak{a} \\
0 & \Lambda / \mathfrak{b}
\end{array}\right)
$$

Right $\Gamma$-modules can be identified with triples $(A, B, f)$ where $A$ is a $\Lambda / \mathfrak{a}$-module, $B$ is a $\Lambda / \mathfrak{b}$-module, and $f: A \otimes_{\Lambda / \mathfrak{a}} \mathfrak{a} \rightarrow B$ is a $\Lambda / \mathfrak{b}$-morphism. More precisely, this identification yields an equivalence of categories (we refer the reader to [4] for more details). We have a functor $\alpha$ : mod- $\Lambda \rightarrow$ mod- $\Gamma$ defined on $\Lambda$-modules $X$ by $\alpha(X)=(X / X \mathfrak{a}, X \mathfrak{a}, \mathfrak{m})$ where $\mathfrak{m}: X / X \mathfrak{a} \otimes_{\Lambda / \mathfrak{a}} \mathfrak{a} \rightarrow X \mathfrak{a}$ is induced by multiplication, and defined on morphisms in the obvious manner. Namely, for $f \in \operatorname{Hom}_{\Lambda}(X, Y), \alpha(f)=\left(\bar{f},\left.f\right|_{X \mathfrak{a}}\right)$, where $\bar{f}$ denotes the induced map $X / X \mathfrak{a} \rightarrow Y / Y \mathfrak{a}$. We shall write $\bmod _{S} \Lambda$ for the full subcategory of mod- $\Lambda$ consisting of those modules with no summand in $\operatorname{add}(S)$, and $\bmod _{S} \Gamma$ will denote the full subcategory of mod- $\Gamma$ consisting of those modules with no summand isomorphic to $(0, T, 0)$ for $T \in \operatorname{add}(S)$. Notice that $\alpha(X) \in \bmod _{S} \Gamma$ for any $\Lambda$-module $X$. The following theorem summarizes the necessary results from [9].

Theorem 4.1 (Martínez Villa [9]). The functor $\alpha: \bmod -\Lambda \rightarrow \bmod _{S} \Gamma$ is full and dense, and induces an equivalence, also denoted $\alpha$, between mod- $\Lambda$ and $\bmod -\Gamma$. Furthermore, the nodes of $\Gamma$ are precisely the $\Gamma$-modules of the form $(T, \overline{0,0)}$ where $\bar{T}$ is a node of $\Lambda$ not isomorphic to any $S_{i}$.

We further recall the fact from [9] that for any $X \in \bmod _{S} \Lambda, X \mathfrak{a}=\tau_{X}(S)$, the trace submodule of $S$ in $X$. As a result, it is clear that

$$
\operatorname{Hom}_{\Lambda}(T, X) \cong \operatorname{Hom}_{\Lambda}(T, X \mathfrak{a})
$$


for any $T \in \operatorname{add}(S)$, and we shall identify these groups where convenient. Also notice, that for $T \in \operatorname{add}(S), \alpha T \cong(T, 0,0)$ is a semisimple injective $\Gamma$-module, while $T_{p}:=(0, T, 0)$ is a semisimple projective $\Gamma$-module. Clearly, for any $T \in \operatorname{add}(S)$, we have

$$
\operatorname{Hom}_{\Gamma}\left(T_{p}, \alpha X\right) \cong \operatorname{Hom}_{\Lambda}(T, X \mathfrak{a}) \cong \operatorname{Hom}_{\Lambda}(T, X)
$$

and using $T \mathfrak{a}=0$,

$$
\operatorname{Hom}_{\Gamma}(\alpha X, \alpha T) \cong \operatorname{Hom}_{\Lambda}(X / X \mathfrak{a}, T) \cong \operatorname{Hom}_{\Lambda}(X, T) .
$$

Again, we make no distinction between a morphism $f$ in any of the above Hom-sets and the corresponding morphism in an isomorphic Hom-set.

Lemma 4.2. Let $0 \rightarrow A \oplus T \stackrel{\left(f_{1}, f_{2}\right)}{\longrightarrow} B \stackrel{g}{\longrightarrow} C \rightarrow 0$ be a short exact sequence in mod- $\Lambda$ with $A, B \in \bmod _{S} \Lambda$ and $T \in \operatorname{add}(S)$. Then $0 \rightarrow \alpha A \oplus T_{p} \stackrel{\left(\alpha f_{1}, f_{2}\right)}{\longrightarrow} \alpha B \stackrel{\alpha g}{\longrightarrow} \alpha C \rightarrow 0$ is a short exact sequence in mod- $\Gamma$. Moreover, if $g$ is right minimal so is $\alpha g$.

Proof. To check that the sequence of $\Gamma$-modules is exact, we must check that the two sequences of $\Lambda$-modules

$$
\begin{aligned}
0 & \rightarrow A / A \mathfrak{a} \stackrel{\bar{f}_{1}}{\longrightarrow} B / B \mathfrak{a} \stackrel{\bar{g}}{\longrightarrow} C / C \mathfrak{a} \rightarrow 0, \\
0 & \rightarrow A \mathfrak{a} \oplus T \stackrel{\left(\left.f_{1}\right|_{\left.\right|_{\mathfrak{a}},} f_{2}\right)}{\longrightarrow} B \mathfrak{a} \stackrel{\left.g\right|_{B \mathfrak{a}}}{\longrightarrow} C \mathfrak{a} \rightarrow 0
\end{aligned}
$$

are exact.

For (4.4), the right exactness of $-\otimes \Lambda / \mathfrak{a}$ implies that the sequence $A / A \mathfrak{a} \oplus T \stackrel{\left(\bar{f}_{1}, \bar{f}_{2}\right)}{\longrightarrow}$ $B / B \mathfrak{a} \stackrel{\bar{g}}{\longrightarrow} C / C \mathfrak{a} \rightarrow 0$ is exact. Since $B \in \bmod _{S} \Lambda, B \mathfrak{a}=\tau_{B}(S)$, and hence $\bar{f}_{2}: T \rightarrow B / B \mathfrak{a}$ is the zero map. Thus to establish the exactness of (4.4) we need only show that $\bar{f}_{1}$ is injective.

Consider $a \in A$ such that $f_{1}(a) \in B \mathfrak{a}=\tau_{B}(S)$. Clearly, $\tau_{B}(S) \in \operatorname{add}(S)$, and thus the submodule $f_{1}(a) \Lambda$ also belongs to $\operatorname{add}(S)$. Since $f_{1}$ is injective, it induces an isomorphism $a \Lambda \stackrel{\cong}{\longrightarrow} f_{1}(a) \Lambda$, from which we conclude that $a \Lambda \in \operatorname{add}(S)$. Clearly, this implies $a \in \tau_{A}(S)=$ $A \mathfrak{a}$ as required.

For (4.5), we only need to check $\left.\operatorname{ker} g\right|_{B \mathfrak{a}} \subseteq f_{1}(A \mathfrak{a})+f_{2}(T)$, as the rest is clear. Given $b \in B \mathfrak{a}$ with $g(b)=0$, there exist $a \in A$ and $t \in T$ such that $b=f_{1}(a)+f_{2}(t)$. Thus $f_{1}(a)=b-f_{2}(t) \in B \mathfrak{a}$, and the previous paragraph yields $a \in A \mathfrak{a}$.

Finally, the statement about minimality follows from the fact that $\alpha$ is full and dense.

We now apply this lemma to study the effect of $\alpha$ on $\mathcal{C}$-resolutions as we did in the last section, where $\mathcal{C}$ again represents a contravariantly finite generator subcategory of mod- $\Lambda$.

Corollary 4.3. Let $X \in \bmod _{S} \Lambda$ with $\Omega_{\mathcal{C}} X \cong Y \oplus T$ for $Y \in \bmod _{S} \Lambda$ and $T \in \operatorname{add}(S)$. Then $\Omega_{\alpha \mathcal{C}}(\alpha X) \cong \alpha Y \oplus T_{p}$.

Proof. Apply the lemma to the exact sequence $0 \rightarrow Y \oplus T \longrightarrow C \stackrel{g}{\longrightarrow} X \rightarrow 0$, where $g$ is a minimal right $\mathcal{C}$-approximation, to get an exact sequence $0 \rightarrow \alpha Y \oplus T_{p} \longrightarrow \alpha C \stackrel{\alpha g}{\longrightarrow} \alpha X \rightarrow 0$ with $\alpha g$ right minimal. Notice that $C \in \bmod _{S} \Lambda$ since any morphism from a node $S_{i}$ to $X$ is not a split monomorphism and hence factors through a projective which is contained in $\mathcal{C}$. From Corollary 3.3 we know that $\alpha g$ is a right $\alpha \mathcal{C}$-approximation of $\alpha X$, and the result follows.

We can now prove the following analogue of Theorem 3.4. 
Proposition 4.4. For any $n \geq 1$ and any $X \in \bmod _{S} \Lambda$, if $\Omega_{\mathcal{C}}^{n}(X)=0$ then $\Omega_{\alpha \mathcal{C}}^{n}(\alpha X)=0$. Furthermore, if $\operatorname{add}(S) \subseteq \mathcal{C}$ then the converse holds as well. In particular, in this case we have $\mathcal{C}-\operatorname{dim} X=\alpha \mathcal{C}-\operatorname{dim} \alpha X$.

Proof. We proceed by induction on $n$, noting that the case $n=1$ is trivial. With the notation of the above lemma, we have $\Omega_{\mathcal{C}}^{n}(X)=\Omega_{\mathcal{C}}^{n-1}\left(\Omega_{\mathcal{C}}(X)\right) \cong \Omega_{\mathcal{C}}^{n-1}(Y \oplus T)=0$. Thus $\Omega_{\mathcal{C}}^{n-1}(Y)=0$, and by induction $\Omega_{\alpha \mathcal{C}}^{n-1}(\alpha Y)=0$. Therefore, for $n \geq 2$,

$$
\Omega_{\alpha \mathcal{C}}^{n}(\alpha X)=\Omega_{\alpha \mathcal{C}}^{n-1}\left(\Omega_{\alpha \mathcal{C}}(\alpha X)\right) \cong \Omega_{\alpha \mathcal{C}}^{n-1}\left(\alpha Y \oplus T_{p}\right) \cong \Omega_{\alpha \mathcal{C}}^{n-1}(\alpha Y)=0,
$$

since $T_{p}$ is projective and thus contained in $\alpha \mathcal{C}$.

Now suppose $\operatorname{add}(S) \subseteq \mathcal{C}$, and $\Omega_{\alpha \mathcal{C}}^{n}(\alpha X)=0$. As above, we induct on $n$, again noting that the case $n=1$ is trivial. For $n \geq 2$ we have

$$
\Omega_{\alpha \mathcal{C}}^{n}(\alpha X)=\Omega_{\alpha \mathcal{C}}^{n-1}\left(\Omega_{\alpha \mathcal{C}}(\alpha X)\right) \cong \Omega_{\alpha \mathcal{C}}^{n-1}\left(\alpha Y \oplus T_{p}\right) \cong \Omega_{\alpha \mathcal{C}}^{n-1}(\alpha Y)=0,
$$

and by induction $\Omega_{\mathcal{C}}^{n-1}(Y)=0$. Thus $\Omega_{\mathcal{C}}^{n}(X) \cong \Omega_{\mathcal{C}}^{n-1}(Y \oplus T) \cong \Omega_{\mathcal{C}}^{n-1}(T)=0$ since $T \in \mathcal{C}$.

Finally, combining this result with our relative homological characterization of representation dimension we can prove, as in the previous section, that $\alpha$ preserves representation dimension.

Corollary 4.5. In the above notation, rep.dim $\Lambda=$ rep.dim $\Gamma$. Consequently, any two stably equivalent Artin algebras have the same representation dimension.

Proof. Let $M$ be an Auslander generator for $\Lambda$. We first show that we may assume that $S$ is a summand of $M$. If $X \in \bmod _{S} \Lambda$, any map from $S$ to $X$ factors through a projective and thus through the right $\operatorname{add}(M)$-approximation $g$ of $X$. Hence $g$ is also a $\operatorname{right} \operatorname{add}(M \oplus S)$ approximation. Meanwhile, if $T \in \operatorname{add}(S), T$ now has a trivial $\operatorname{add}(M \oplus S)$-resolution. This shows that for any $X,(M \oplus S) \operatorname{dim} X \leq M-\operatorname{dim} X$. Since $M$ is an Auslander generator, $M \oplus S$ must be one as well.

Recall that the indecomposable projective $\Gamma$-modules are precisely the modules $\alpha P$ or $\left(S_{i}\right)_{p}$ for an indecomposable projective $P_{\Lambda}$ or a node $S_{i}$, while the indecomposable injective $\Gamma$-modules are precisely those modules of the form $\alpha I$ or $\alpha S_{i}$ for an indecomposable injective $\Lambda$-module $I$ or a node $S_{i}$. Hence $\alpha M \oplus S_{p}$ is a generator-cogenerator for $\Gamma$, and any generatorcogenerator for $\Gamma$ has this form (where $M_{\Lambda}$ is a generator-cogenerator with $S \in \operatorname{add}(M)$ ). In view of the preceding result, $\Lambda$ and $\Gamma$ must have equal representation dimensions.

Of course, the second statement follows now from Corollary 3.5 and the fact that any stable equivalence can be factored as a (possibly trivial) separation of nodes, composed with a stable equivalence between algebras without nodes, and then with the inverse of a (possibly trivial) separation of nodes.

\section{The Case of Representation Dimension 3}

In order to indicate how relative homological invariants of stable equivalence may relate to other properties of algebras, we now restrict our attention to a self-injective Artin algebra $\Lambda$ of representation dimension three. For self-injective algebras, the condition of $\Lambda$ having no nodes is equivalent to $\Lambda$ having no blocks of Loewy length two, or even to $\Lambda$ having no indecomposable projective modules of length two. We thus assume that $\Lambda$ has no blocks of Loewy length two or smaller, and fix an Auslander generator $M=M^{\prime} \oplus \Lambda$ where $M^{\prime} \in$ $\bmod _{\mathcal{P}} \Lambda$. 
The focus of this section shall be to relate the number $n_{\Lambda}$ of isomorphism classes of simple $\Lambda$-modules to the minimal number $m_{\Lambda}$ of nonisomorphic, indecomposable, nonprojective summands of an Auslander generator in this case. Notice that the latter is invariant under stable equivalence by Corollary 3.6. Our starting point will be a result of Rouquier [12], stating that $m_{\Lambda}$ is at least half $n_{\Lambda}$. We will show that "half" may be replaced with "twothirds", and in case $\Lambda$ is weakly symmetric or of Loewy length three, $M^{\prime}$ has at least $n_{\Lambda}$ nonisomorphic indecomposable summands. We begin by citing the following lemma from $[12]$.

Lemma 5.1. Let $0 \rightarrow M_{1} \longrightarrow M_{0} \longrightarrow S \rightarrow 0$ be a minimal $M$-resolution for the simple $\Lambda$ module $S$. Then, either $M_{1}, M_{0} \in \operatorname{add}\left(M^{\prime}\right) \subseteq \bmod _{\mathcal{P}} \Lambda$ or $M_{0}$ is isomorphic to the projective cover of $S$ and $M_{1} \cong \Omega S$.

For any additive subcategory $\mathcal{C} \subseteq \bmod -\Lambda$, let $\langle\mathcal{C}\rangle \leq K_{0}(\Lambda)$ denote the subgroup of the Grothendieck group generated by the objects of $\mathcal{C}$. If $X$ is a $\Lambda$-module, we will write $[X]$ for the image of $X$ in $K_{0}(\Lambda)$.

Proposition 5.2. In the above notation, $m_{\Lambda}$ is greater than or equal to $2 n_{\Lambda} / 3$.

Proof. Let $k$ equal the number of simple modules $S$ such that $[S] \in\left\langle\operatorname{add}\left(M^{\prime}\right)\right\rangle$, and let $m$ denote the number of indecomposable summands of $M^{\prime}$. Clearly, $m \geq \operatorname{rk}\left\langle\operatorname{add}\left(M^{\prime}\right)\right\rangle \geq k$. By the above lemma, if $S$ is a simple module such that $[S] \notin\left\langle\operatorname{add}\left(M^{\prime}\right)\right\rangle, \Omega S \mid M^{\prime}$ (where $A \mid B$ means that $A$ is isomorphic to a direct summand of $B$ ). Consequently, $S \mid \Omega^{-1} M^{\prime}$, and $\Omega^{-1} M^{\prime}$ has at least $n_{\Lambda}-k$ simple summands. Furthermore, we know that $\Omega^{-1} M^{\prime} \oplus \Lambda$ is again an Auslander generator and thus the previous lemma guarantees $\Omega T \mid \Omega^{-1} M^{\prime}$ for any simple module $T$ with $[T] \notin\left\langle\operatorname{add}\left(\Omega^{-1} M^{\prime}\right)\right\rangle$. Letting $l$ equal the number of such $T$, we have $m \geq \operatorname{rk}\left\langle\operatorname{add}\left(\Omega^{-1} M^{\prime}\right)\right\rangle \geq n_{\Lambda}-l$. On the other hand, since $\Lambda$ is assumed to have no blocks of Loewy length 2 , none of the modules $\Omega T$ can be simple when $T$ is simple (this follows from Lemma 4.3 of [3], for example). Thus, counting summands of $\Omega^{-1} M^{\prime}$, we have $m \geq n_{\Lambda}-k+l$. Altogether we have the three inequalities $m \geq k, m \geq n_{\Lambda}-l$ and $m \geq n_{\Lambda}-k+l$, and adding them together yields $3 m \geq 2 n_{\Lambda}$ or $m \geq 2 n_{\Lambda} / 3$ as required.

Notice that if the simple $\Lambda$-modules are not periodic, there is some $i$ such that $\Omega^{i-1} M^{\prime}$ has no simple summands, and hence $k=n_{\Lambda}$ in the above notation with respect to the Auslander generator $\Omega^{i} M^{\prime} \oplus \Lambda$. In other words, $\left\langle\operatorname{add}\left(\Omega^{i} M^{\prime}\right)\right\rangle=K_{0}(\Lambda)$, and we have $m_{\Lambda} \geq n_{\Lambda}$. By Proposition 1.4 of [11], this periodicity condition is satisfied by self-injective algebras of Loewy length three and infinite representation type, which necessarily have representation dimension three as well. We state this as a corollary.

Corollary 5.3. If $\Lambda$ has no periodic simple modules-for instance, if $\Lambda$ is self-injective of Loewy length three and infinite representation type-then $m_{\Lambda} \geq n_{\Lambda}$.

We will construct an example in the next section to show that $\operatorname{add}\left(M^{\prime}\right)$ does not necessarily generate the entire Grothendieck group $K_{0}(\Lambda)$. However, the following results suggest that the number of indecomposable summands of $M^{\prime}$ will often be much larger than the above lower bound. In particular, it would be interesting to determine whether $m_{\Lambda} \geq n_{\Lambda}$ always holds. We begin with some preliminary results which will prove useful in investigating some consequences of the existence of a simple module $S$ such that $[S] \notin\left\langle\operatorname{add}\left(M^{\prime}\right)\right\rangle$.

Proposition 5.4. Let $N$ be an indecomposable $\Lambda$-module such that $[N] \notin\left\langle\operatorname{add}\left(M^{\prime}\right)\right\rangle$, and suppose that $\underline{\operatorname{Hom}}_{\Lambda}\left(M^{\prime}, N^{\prime}\right)=0$ for all proper submodules $N^{\prime}$ of $N$. Then $\underline{\operatorname{Hom}}_{\Lambda}\left(M^{\prime}, N\right)=$ 0. 
Proof. Consider a map $f: M^{\prime} \rightarrow N$. If $f$ is not epimorphic, $f$ factors through a proper submodule of $N$ and thus through a projective module by hypothesis. If $f$ is an epimorphism, any map $g: P \rightarrow N$ with $P$ projective factors through $f$. In particular, given a minimal $M$-resolution $0 \rightarrow M_{1} \longrightarrow M_{0} \oplus P_{0} \stackrel{(u v)}{\longrightarrow} N \rightarrow 0$ for $N$ where $M_{0}, M_{1} \in \bmod _{\mathcal{P}} \Lambda$ and $P_{0}$ is projective, $v: P_{0} \rightarrow N$ must factor through $f$, and hence $(u f): M_{0} \oplus M^{\prime} \rightarrow N$ is epimorphic as is $(M,(u f)):\left(M, M_{0} \oplus M^{\prime}\right) \rightarrow(M, N)$. Thus $(u f)$ is a $\operatorname{right} \operatorname{add}(M)$-approximation of $N$, and therefore $M_{0} \oplus M^{\prime}$ contains a direct summand isomorphic to $M_{0} \oplus P_{0}$. Since $M_{0}, M^{\prime} \in \bmod _{\mathcal{P}} \Lambda$, we must have $P_{0}=0$. However, then $[N]=\left[M_{0}\right]-\left[M_{1}\right] \in\left\langle\operatorname{add}\left(M^{\prime}\right)\right\rangle$, contrary to our assumption. Thus $f$ cannot be an epimorphism and $\underline{\operatorname{Hom}}_{\Lambda}\left(M^{\prime}, N\right)=0$.

Lemma 5.5. Suppose $\operatorname{Hom}_{\Lambda}\left(M^{\prime}, N\right)=0$ for an indecomposable $\Lambda$-module $N$. Then the short exact sequence $0 \rightarrow \Omega N \longrightarrow P_{N} \stackrel{\pi}{\longrightarrow} N \rightarrow 0$, where $P_{N}$ is the projective cover of $N$, is an $M$-resolution for $N$. In particular, $\Omega N \mid M^{\prime}$.

Proof. Let $0 \rightarrow M_{1} \longrightarrow M_{0} \stackrel{h}{\longrightarrow} N \rightarrow 0$ be an $M$-resolution of $N$. The stated assumptions imply that $(M, \pi):\left(M, P_{N}\right) \rightarrow(M, N)$ is surjective, and thus it suffices to check that $\Omega N \in \operatorname{add}(M)$. Since $(M, h):\left(M, M_{0}\right) \rightarrow(M, N)$ is also surjective, and $M_{0}, P_{N} \in \operatorname{add}(M)$, $h$ factors through $\pi$ and $\pi$ factors through $h$. Since $\pi$ is clearly right minimal, $P_{N}$ splits off as a direct summand of $M_{0}$ and it follows that $\Omega N \mid M_{1}$. Since $\Omega N$ is indecomposable and $M_{1} \in \operatorname{add}\left(M^{\prime}\right), \Omega N \mid M^{\prime}$.

We can now establish some significant consequences of the existence of a simple module $S$ for which $[S] \notin\left\langle\operatorname{add}\left(M^{\prime}\right)\right\rangle$.

Theorem 5.6. Suppose that $S$ is a simple $\Lambda$-module with $[S] \notin\left\langle\operatorname{add}\left(M^{\prime}\right)\right\rangle$, and let $N$ be any $\Lambda$-module with soc $N \cong S$. Then the following hold.

(1) $N$ is uniserial.

(2) $\underline{\operatorname{Hom}}_{\Lambda}\left(M^{\prime}, N\right)=0$.

(3) $\Omega N \mid M^{\prime}$.

(4) $[N] \notin\left\langle\operatorname{add}\left(M^{\prime}\right)\right\rangle$.

Proof. We proceed by induction on the Loewy length $r$ of $N$. If $r=1, N$ must be isomorphic to $S$ and (2) and (3) follow from the previous 2 results while (1) and (4) are trivial. As our inductive hypothesis we shall assume that properties (1) through (4) hold whenever $N$ is any $\Lambda$-module of Loewy length at most $r$ and with socle isomorphic to $S$. Now suppose that $N$ has Loewy length $r+1$ and $\operatorname{soc} N \cong S$. There exists a direct sum decomposition $N / N J=T_{1} \oplus \cdots \oplus T_{s}$ for simple $\Lambda$-modules $T_{i}$, and for each $i$ we can find a submodule $N_{i} \subseteq N$ such that $N J \subset N_{i}$ and $N_{i} / N J=T_{i}$. Clearly we have $N=\sum_{i=1}^{s} N_{i}$ and $N_{i} \cap N_{j}=N J$ for all $i \neq j$.

Furthermore, we have non-split exact sequences $0 \rightarrow N J \longrightarrow N_{i} \longrightarrow T_{i} \rightarrow 0$ which show that $\underline{\operatorname{Hom}}_{\Lambda}\left(\Omega T_{i}, N J\right) \cong \operatorname{Ext}_{\Lambda}^{1}\left(T_{i}, N J\right) \neq 0$. Since the induction hypothesis yields $\underline{\operatorname{Hom}}_{\Lambda}\left(M^{\prime}, N J\right)=0$, we conclude that $M^{\prime}$ has no direct summand isomorphic to $\Omega T_{i}$ for any $i$. Consequently, by Proposition 5.4 and Lemma 5.5, we must have $\left[T_{i}\right] \in\left\langle\operatorname{add}\left(M^{\prime}\right)\right\rangle$ for each $i$. Since $[N J] \notin\left\langle\operatorname{add}\left(M^{\prime}\right)\right\rangle$ by the induction hypothesis, neither is $[N]=[N J]+\left[T_{1}\right]+$ $\cdots+\left[T_{s}\right]$, and we have established (4) for $N$.

To prove that $\underline{\operatorname{Hom}}_{\Lambda}\left(M^{\prime}, N\right)=0$, it now suffices, by Proposition 5.4, to show that $\underline{\operatorname{Hom}}_{\Lambda}\left(M^{\prime}, N^{\prime}\right)=0$ for all proper submodules $N^{\prime}$ of $N$. Suppose that this is not the case, and choose a submodule $N^{\prime}$ of minimal length for which $\underline{\operatorname{Hom}}_{\Lambda}\left(M^{\prime}, N^{\prime}\right) \neq 0$. From the inductive hypothesis, it is immediate that the Loewy length of $N^{\prime}$ must be $r+1$. Then 
$N^{\prime} J \subseteq N J$ and both of these modules have Loewy length $r$. Since $N J$ is uniserial, we must have $N^{\prime} J=N J$. Thus

$$
\left[N^{\prime}\right]=[N J]+\left[T_{i_{1}}\right]+\cdots\left[T_{i_{t}}\right] \notin\left\langle\operatorname{add}\left(M^{\prime}\right)\right\rangle,
$$

and the minimality of $N^{\prime}$ allows us to invoke Proposition 5.4, which yields the contradiction $\underline{\operatorname{Hom}}_{\Lambda}\left(M^{\prime}, N^{\prime}\right)=0$. This proves (2), and (3) now follows via Lemma 5.5.

Finally, we must show that $N$ is uniserial. Clearly it suffices to show that $s=1$ so that $N / N J$ is simple. However, if $s \geq 2$ we would have a non-split exact sequence

$$
0 \rightarrow N J \longrightarrow N_{1} \oplus \sum_{i=2}^{s} N_{i} \longrightarrow N \rightarrow 0,
$$

yielding $\underline{\operatorname{Hom}}_{\Lambda}(\Omega N, N J) \cong \operatorname{Ext}_{\Lambda}^{1}(N, N J) \neq 0$. By (3) we would thus have $\underline{\operatorname{Hom}}_{\Lambda}\left(M^{\prime}, N J\right) \neq$ 0 , but this contradicts the induction hypothesis.

Remark. Working with $M$-coresolutions instead of $M$-resolutions leads to natural duals of the previous three results. Their explicit formulation is straight-forward and thus left to the reader. However, we shall incorporate the dual of the preceding theorem freely in the following corollaries.

Corollary 5.7. If $S$ is a simple $\Lambda$-module such that $[S] \notin\left\langle\operatorname{add}\left(M^{\prime}\right)\right\rangle$, then both the injective envelope and the projective cover of $S$ are uniserial. Furthermore, if $N$ is any submodule of the injective envelope of $S$ and $N^{\prime}$ is any quotient of the projective cover of $S$, then $\Omega N \mid M^{\prime}$ and $\Omega^{-1} N^{\prime} \mid M^{\prime}$.

We can strengthen this result even further by considering the Nakayama automorphism $\mathcal{N}$ of $\Lambda$. Recall that $\mathcal{N}$ : mod- $\Lambda \rightarrow \bmod -\Lambda$ is the functor $D H_{0}(-, \Lambda)$, which is an equivalence when $\Lambda$ is self-injective. If $S$ is a simple $\Lambda$-module, $\mathcal{N} S$ is isomorphic to the top of the injective hull of $S$ and hence is also simple. The permutation that $\mathcal{N}$ induces on the set of isomorphism classes of simple modules is known as the Nakayama permutation.

Since $\mathcal{N}$ is an equivalence, it is clear that $\mathcal{N} M$ is an Auslander generator for $\Lambda$ whenever $M$ is. It is also not hard to see that $\mathcal{N}$ induces an automorphism of $K_{0}(\Lambda)$ by $\mathcal{N}[X]=[\mathcal{N} X]$. Thus $[S] \notin\left\langle\operatorname{add}\left(M^{\prime}\right)\right\rangle$ if and only if $[\mathcal{N} S] \notin\left\langle\operatorname{add}\left(\mathcal{N} M^{\prime}\right)\right\rangle$. Of course, if this is the case, we can apply Corollary 5.7 to the simple module $\mathcal{N} S$ and the Auslander generator $\mathcal{N} M$ to conclude that the projective cover and injective hull of $\mathcal{N} S$ are also uniserial. We state this result as another corollary.

Corollary 5.8. If $S$ is a simple $\Lambda$-module such that $[S] \notin\left\langle\operatorname{add}\left(M^{\prime}\right)\right\rangle$ and $T$ is any simple module in the $\mathcal{N}$-orbit of $S$, then both the injective envelope and the projective cover of $T$ are uniserial.

Corollary 5.9. If $\Lambda$ is weakly symmetric, then $\left\langle\operatorname{add}\left(M^{\prime}\right)\right\rangle=K_{0}(\Lambda)$, and hence $M$ has at least $n_{\Lambda}$ nonprojective indecomposable summands.

Proof. Suppose $[S] \notin\left\langle\operatorname{add}\left(M^{\prime}\right)\right\rangle$ for some simple module $S$, and let $I$ be the injective envelope of $S$. It follows from the proof of Theorem 5.6 that any composition factor $T$ of $I / \operatorname{soc} I$ satisfies $[T] \in\left\langle\operatorname{add}\left(M^{\prime}\right)\right\rangle$. But since $\Lambda$ is weakly symmetric, $S \cong \mathcal{N} S \cong I / I J$ and thus $[S] \in\left\langle\operatorname{add}\left(M^{\prime}\right)\right\rangle$, a contradiction.

For our final result, we further restrict our attention to a self-injective algebra $\Lambda$ of Loewy length three. Tang has shown in [13] that any $\Lambda^{\prime}$ stably equivalent to such a $\Lambda$ has the same number of (nonprojective) simple modules as $\Lambda$, provided that the separated quiver of $\Lambda$ has 
more than two connected components. Using the fact that self-injective algebras of Loewy length three have representation dimension at most three, we can obtain a much weaker result, but without any condition on the separated quiver of $\Lambda$.

Proposition 5.10. Suppose $\Lambda$ and $\Lambda^{\prime}$ are stably equivalent self-injective algebras and that $\Lambda$ has Loewy length three and no blocks of smaller Loewy length. Then $n_{\Lambda^{\prime}} \leq 3 n_{\Lambda}$. Furthermore, if $\Lambda^{\prime}$ is weakly symmetric (e.g. if $\Lambda$ is symmetric) or of Loewy length three, $n_{\Lambda^{\prime}} \leq 2 n_{\Lambda}$.

Proof. From [1] we know that rep.dim $\Lambda \leq 3$. Since the result is of course true for algebras of finite representation type, we may assume that rep.dim $\Lambda=3$. Moreover, $\Lambda \oplus \Lambda / J \oplus \Lambda / J^{2}$ is an Auslander generator with $2 n_{\Lambda}$ nonprojective indecomposable summands. By Theorem $3.4, \Lambda^{\prime}$ also has representation dimension three and an Auslander generator with $2 n_{\Lambda}$ nonprojective indecomposable summands. From Proposition 5.2 and Corollaries 5.3 and 5.9 we now conclude that $\Lambda^{\prime}$ has at most $3 n_{\Lambda}$ isomorphism classes of simple modules, or at most $2 n_{\Lambda}$ if $\Lambda^{\prime}$ is weakly symmetric or of Loewy length three. Furthermore, we note that if $\Lambda$ is symmetric, then $\mathcal{N} X \cong X$ for every $\Lambda$-module $X$ and thus the same holds for $\Lambda^{\prime}$, as it follows from [3] that $\mathcal{N} \cong \Omega^{-2} D T r$ commutes with any stable equivalence. In particular, $\mathcal{N} S \cong S$ for every simple $\Lambda^{\prime}$-module $S$, and thus $\Lambda^{\prime}$ is weakly symmetric when $\Lambda$ is symmetric.

\section{An EXAmple}

The main purpose of this section is to present an example of a self-injective algebra $\Lambda$ of representation dimension three with a simple module $S$ and an Auslander generator $M=M^{\prime} \oplus \Lambda$ such that $[S] \notin\left\langle\operatorname{add}\left(M^{\prime}\right)\right\rangle$. In order to verify that the module $M$ is indeed an Auslander generator we first prove a general criterion for checking this in a finite number of steps. Recall that since add $(M)$ is a contravariantly finite subcategory of mod- $\Lambda$, each indecomposable summand of $M$ is the target of a relative sink map in $\operatorname{add}(M)$, i.e., a minimal right almost-split morphism in $\operatorname{add}(M)$.

Proposition 6.1. Suppose that $M_{1}, \ldots, M_{m}$ are nonisomorphic indecomposable $\Lambda$-modules, such that $M=\bigoplus_{i=1}^{m} M_{i}$ is a non-semisimple generator-cogenerator for $\Lambda$. If $K_{i}$ denotes the kernel of the relative sink map $f_{i}: N_{i} \rightarrow M_{i}$ in $\operatorname{add}(M)$, then

$$
\text { gl.dim } \operatorname{End}_{\Lambda}(M)=\sup \left\{\operatorname{add}(M)-\operatorname{dim} K_{i}\right\}_{i=1}^{m}+2 .
$$

Proof. Let $\Gamma=\operatorname{End}_{\Lambda}(M)$. Then gl.dim $\Gamma=\sup \left\{\operatorname{pd} S_{i}\right\}_{i=1}^{m}$ where $S_{i}$ is the simple $\Gamma$-module corresponding to the indecomposable projective $\Gamma$-module $\left(M, M_{i}\right)$. Moreover, $\left(M, N_{i}\right) \stackrel{\left(M, f_{i}\right)}{\longrightarrow}\left(M, M_{i}\right) \rightarrow S_{i} \rightarrow 0$ is a minimal projective presentation for $S_{i}$ over $\Gamma$, and hence pd $S_{i}=\operatorname{pd}\left(M, K_{i}\right)+2$. But, as observed in Section 3, pd $\left(M, K_{i}\right)=\operatorname{add}(M)-\operatorname{dim} K_{i}$, and the proof is complete.

Thus, in order to find the global dimension of the endomorphism ring of a given generatorcogenerator $M$, we need only compute the relative sink maps in $\operatorname{add}(M)$ to each indecomposable summand of $M$, and then compute the minimal add $(M)$-resolutions of the kernels of these sink maps. In particular, if we know the representation dimension of $\Lambda$, we can determine whether $M$ is an Auslander generator in a finite number of steps. While this method suffices to verify the following example, it does not in general lead to an efficient algorithm for finding Auslander generators as the necessary computations vary substantially along with the choice of the module $M$ being tested. 
We define our algebra $\Lambda$ as a quotient of the path algebra (over an infinite field $k$ ) of the quiver

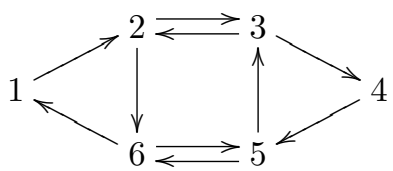

such that the indecomposable projective left $\Lambda$-modules have graphs:

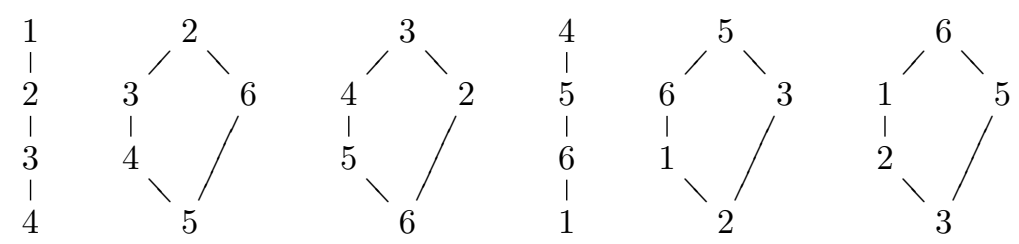

Observe that $\Lambda$ is a self-injective special biserial algebra. Furthermore $\Lambda$ has infinite representation type, as the following quiver representations correspond to pairwise nonisomorphic, indecomposable $\Lambda$-modules for each possible $c \in k$.

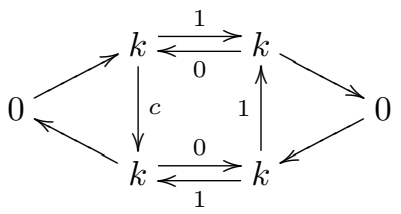

Hence, by [6] we know that rep.dim $\Lambda=3$, and that $\Lambda$ has an Auslander generator $N$ consisting of the direct sum of all the indecomposable projectives, all the uniserial modules, and all the modules of the form $P / \operatorname{soc} P$ or $\operatorname{rad} P$ for an indecomposable projective $P$. The module $N$ thus has 6 indecomposable projective summands and 30 indecomposable nonprojective summands, whose graphs the reader may easily write down.

Our goal is to construct another Auslander generator $M=\Lambda \oplus M^{\prime}$ such that no indecomposable summand of $M^{\prime}$ has the simple module $S_{1}$ as a composition factor. Clearly, we must then have $\left[S_{1}\right] \notin\left\langle\operatorname{add}\left(M^{\prime}\right)\right\rangle$, and Corollary 5.7 gives the necessary condition that the modules

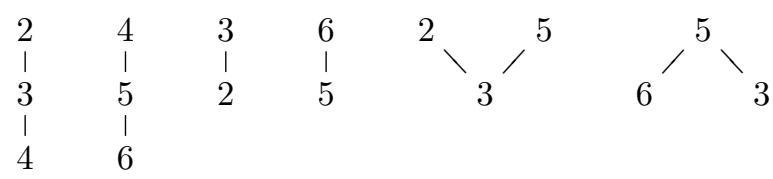

are summands of $M^{\prime}$. Thus, we define $M^{\prime}$ to be the direct sum of all the indecomposable nonprojective summands of $N$ that do not have $S_{1}$ as a composition factor together with the last two modules in the list above (the first four are already summands of $N$ ). It is now possible to check that gl.dim $\operatorname{End}_{\Lambda}\left(\Lambda \oplus M^{\prime}\right)=3$ with the help of Proposition 6.1. We omit the lengthy calculations that include computing all 28 relative sink maps in $\operatorname{add}(M)$, finding the minimal right $\operatorname{add}(M)$-approximations of the kernels of each sink map, and finally checking that the kernels of these $\operatorname{add}(M)$-approximations all belong to $\operatorname{add}(M)$.

We conclude by noting an additional interesting property of this Auslander generator $M$. If we take the syzygies of the six modules listed above which we know have to be summands of $M^{\prime}$, we see that $P_{4} / \operatorname{soc} P_{4}$ and $S_{1}$ must be summands of $\Omega M^{\prime}$. It now follows from Theorem $5.6(2)$ and Corollary 5.7 that $\left\langle\operatorname{add}\left(\Omega M^{\prime}\right)\right\rangle=K_{0}(\Lambda)$. Hence, this example still leaves open the possibility that if $M=\Lambda \oplus M^{\prime}$ is an Auslander generator for a self-injective algebra $\Lambda$ of representation dimension three, then $\left\langle\operatorname{add}\left(\Omega^{i} M^{\prime}\right)\right\rangle=K_{0}(\Lambda)$ for some $i$. If this 
were true, it would establish that the number of indecomposable summands of $M^{\prime}$ is at least the number of simple $\Lambda$-modules.

\section{REFERENCES}

[1] M. Auslander. Representation dimension of Artin algebras. Queen Mary College mathematical notes, University of London, 1971.

[2] M. Auslander and I. Reiten. Stable equivalence of Artin algebras. Proc. of the Conf. on Orders, Group Rings and Related Topics (Ohio State Univ., Columbus, Ohio, 1972), 8-71, Lecture Notes in Math., Vol. 353, Springer, Berlin, 1973.

[3] M. Auslander and I. Reiten. Representation theory of Artin algebras VI. Comm. Algebra 6 (1978), no. $3,267-300$.

[4] M. Auslander, I. Reiten, S. Smalo. Representation theory of Artin algebras. Cambridge Studies in Advanced Mathematics 36, Cambridge Univ. Press, 1995.

[5] M. Auslander, O. Solberg. Relative homology and representation theory I. Comm. Algebra 21 (1993), no. 9, 2995-3031.

[6] K. Erdmann, T. Holm, O. Iyama, J. Schroer. Radical embeddings and representation dimension. Adv. Math. 185 (2004), no. 1, 159-177.

[7] X. Guo. Representation dimension: an invariant under stable equivalence. Trans. AMS 357 (2005), no. 8, 3265-3284.

[8] O. Iyama. Finiteness of representation dimension. Proc. AMS 131 (2002), no. 4, 1011-1014.

[9] R. Martínez Villa. Algebras stably equivalent to l-hereditary. Representation theory II (Proc. ICRA II, Carleton Univ., Ottawa, Ont., 1979), 396-431, Lecture Notes in Math., 832, Springer, Berlin, 1980.

[10] R. Martínez Villa. Properties that are left invariant under stable equivalence. Comm. Algebra 18 (1990), no. $12,4141-4169$.

[11] R. Martínez Villa. Applications of Koszul algebras: the preprojective algebra. Representation theory of algebras (Cocoyoc, 1994), 487-504, CMS Conf. Proc. 18, Amer. Math. Soc., Providence, RI, 1996.

[12] R. Rouquier. Dimensions of triangulated categories. Preprint (2004).

[13] A. Tang. A note on the stable equivalence conjecture. Comm. Algebra 24 (1996), no. 9, 2793-2809.

[14] C. Xi. Representation dimension and quasi-hereditary algebras. Adv. Math. 168 (2002), no. 2, 193-212.

Department of Mathematics, University of California, Berkeley, CA 94720, USA

E-mail address: asdugas@math.berkeley.edu 